Revista Mexicana de Economía y Finanzas Nueva Época, Vol. 14 No. 1, (2019), pp. 57-77

DOI: http://dx.doi.org/10.21919/remef.v14i1.359

\title{
Desarrollo de un modelo Logit para examinar el comportamiento del ahorro en la región centro de México, de acuerdo al perfil de los hogares
}

\author{
Adriana Helena Cruz León ${ }^{1}$ \\ Instituto Politécnico Nacional, México \\ Distinción Especial Categoría Tesis, XXXIV Premio de Investigación Financiera IMEF-EY 2018 \\ José Carlos Trejo García ${ }^{2}$ \\ Instituto Politécnico Nacional, México \\ Humberto Ríos Bolívar ${ }^{3}$ \\ Instituto Politécnico Nacional, México \\ (Recepción: 27/abril/2018, aceptado: 22/agosto/2018)
}

\begin{abstract}
Resumen
Esta investigación tiene como objetivo examinar el comportamiento del ahorro en hogares de la Región Centro de México (SEDATU, 2015) considerando su perfil socioeconómico. Se proponen perfiles del hogar (Cruz, 2017) utilizando árboles de decisión y se calcula la probabilidad de ahorro (ENIGH, 2014) con un modelo logit. En los resultados del modelo, se encontró que las variables son significativas y se determina la probabilidad del nivel de ahorro de los hogares. A diferencia de la LCH (Modigliani, 1963), se recomienda identificar perfiles de ingreso y gasto con una segmentación de la población más allá de grupos de edad del jefe de familia. En determinados grupos de edad se aprecia que los de menor escolaridad tienen una mayor proporción de hogares con posibilidad de ahorrar; este comportamiento se limita a una investigación posterior para estudiar el efecto de otras variables. El encuadre del entorno regional contribuye a analizar con un mayor nivel de especificidad el comportamiento de ahorro, de acuerdo al perfil del hogar. Finalmente, se muestra que, con las variables propuestas, aumenta la probabilidad de ahorro en el hogar.

Clasificación JEL: E21, D1, C44, D14

Palabras clave: Ahorro, Comportamiento, Decisión, Finanzas Personales
\end{abstract}

\section{Development of A Logit Model to Examine the Behavior of Savings in the Central Region of Mexico, According to the Profile of Households}

\section{Abstract}

The objective of this research is to examine the behavior of savings in households in the Central Region of Mexico (SEDATU, 2015) considering their socioeconomic profile. Household profiles are proposed (Cruz, 2017) using decision trees and the probability of saving is calculated (ENIGH, 2014) with a logit model. In the results of the model, it was found that the variables are significant and the probability of the saving level of the households is determined. Unlike the LCH (Modigliani, 1963), it is recommended to identify income and expenditure profiles with a segmentation of the population beyond the age groups of the head of the family. In certain age groups, it can be seen that those with less education have a higher proportion of households with the possibility of saving; this behavior is limited to a subsequent investigation to study the effect of other variables.

\footnotetext{
${ }^{1}$ M. en C. SEPI-ESE del Instituto Politécnico Nacional, mail: ahcruzl@hotmail.com

${ }^{2}$ Profesor Investigador, Coordinador de Posgrado, SNI I, en SEPI-ESE del Instituto Politécnico Nacional, mail: jtrejog@ipn.mx

${ }^{3}$ Profesor Investigador, SNI III, en SEPI-ESE del Instituto Politécnico Nacional, mail: hrios@ipn.mx
} 
REMEF (The Mexican Journal of Economics and Finance)

\section{Abstract}

The framing of the regional environment contributes to analyzing with a higher level of specificity the saving behavior, according to the household profile. Finally, it is shown that, with the proposed variables, the probability of saving at home increases.

JEL Classification: E21, D1, C44, D14

Keywords: Saving, Behavior, Decision, Personal Finance

\section{Introducción}

La cultura del ahorro en México no es generalizada, específicamente en lo referente al ahorro voluntario el crecimiento ha sido limitado en el país, esto puede apreciarse al analizar la composición de las fuentes de recursos financieros como porcentaje del PIB en donde del 2008 al 2011 la parte correspondiente al ahorro voluntario ha pasado del $39.12 \%$ al $38.87 \%$ (Góngora, 2013), en línea con lo anterior y de acuerdo con la Encuesta Nacional de Inclusión Financiera, para el 2015 solamente el $38 \%$ de los adultos en México ahorraban formalmente (Consejo Nacional de Inclusión Financiera, 2017). Esta situación lleva a que la economía en su conjunto esté sujeta a una mayor vulnerabilidad (por ejemplo, el aumento en las perturbaciones financieras en los países en desarrollo que provoca movimientos de capital entre países) además que la población no pueda acceder a los beneficios fundamentales del ahorro, por ejemplo: recursos para el retiro, afrontar imprevistos, la inversión y por último la acumulación de riqueza. Esta situación hace necesario tener una fuente de información detallada y confiable sobre la conducta de ahorro de los hogares para el diseño de estrategias de captación.

La presente investigación es una extensión al modelo nacional presentado en el trabajo (Cruz, 2017), del cual se retoma, por un lado, la definición propuesta de perfiles de los hogares a efecto de verificar la capacidad explicativa de los mismos para un análisis regional, y por otro, las variables generales definidas para estudiar la probabilidad de ahorro de los hogares. El encuadre de la investigación en un entorno regional contribuye a analizar con un mayor nivel de especificidad el comportamiento de ahorro de los hogares, caracterizándolos de acuerdo a su perfil. Como hipótesis de la investigación se plantea que el nivel del ahorro depende del perfil del hogar y de variables que afectan la incertidumbre en el ingreso y no solo por el nivel de ingreso del mismo.

Con el objetivo de examinar el comportamiento del ahorro de los hogares específicamente en la región centro del país de acuerdo a su perfil socioeconómico, nivel de ingreso y factores de incertidumbre, la presente investigación desarrolla un modelo logit. Al respecto de los factores de incertidumbre, la presente investigación adiciona al trabajo citado la variable de subempleo del jefe de familia (persona que trabaja menos de 35 horas a la semana) con el propósito de incorporar el efecto de la precarización del empleo en conjunto con la incidencia de desempleo, ambas variables son reconocidas por su influencia en la reducción de los ingresos de los individuos, así como el incremento en la pobreza (Organización para la Cooperación y el Desarrollo Económico, 2017).

Se considera el perfil del hogar de acuerdo a las características del jefe de familia: edad, estado civil, escolaridad, género, además de la presencia de menores de 12 años; dentro de las variables que influyen en la incertidumbre en el ingreso se considera el estado de salud del jefe de familia, si se ocupa en autoempleo, el enfrentar una situación reciente de desempleo, ser subempleado y la cantidad de personas que reciben un ingreso monetario en el hogar. Adicionalmente se considera, como parte del análisis, el ingreso corriente además de la propiedad de una vivienda.

Se toma en cuenta la clasificación en tres regiones propuesta por la Secretaría de Desarrollo Agrario, Territorial y Urbano (2015): norte, centro, sur. Presentando los resultados del modelo para la Región Centro que comprende los estados de Nayarit, Jalisco, Colima, 
Michoacán, Zacatecas, Aguascalientes, San Luis Potosí, Guanajuato, Querétaro, Hidalgo, Tlaxcala, Morelos, Estado de México y Ciudad de México. Incorporar la consideración regional propuesta por la SEDATU permite presentar resultados de acuerdo con las regiones propuestas en los Programas Regionales de Desarrollo, además de que, como lo reconoce (Gasca, 2009), el estudio de las regiones y lugares permite dar un significado práctico y aplicado a la regionalización de los procesos económicos y entender los distintos procesos relacionados con la territorialidad de los individuos.

La investigación está estructurada en cuatro apartados: en el primero se presentan las principales teorías consideradas para el estudio del ahorro, aportaciones de la Economía Conductual y una revisión de los trabajos empíricos que estudian específicamente la problemática de México; en el segundo apartado se detallan los aspectos metodológicos considerados para el selección de variables y diseño de perfiles de ahorro; en la tercer sección se presenta el modelo econométrico de probabilidad de ahorro de los hogares y en el último apartado se muestran las conclusiones del trabajo que destacan los hallazgos principales del modelo y el contraste de lo predicho por la Teoría Prospectiva (rama de la Economía Conductual) en lo referente a las variables de incertidumbre.

\section{Antecedentes teóricos y empíricos}

Se identifican tres enfoques teóricos principales que se han encargado del estudio del ahorro, visto éste como un elemento que permite transferir ingreso de un periodo a otro para mantener una senda de consumo ante la presencia de diversas afectaciones (provenientes de la riqueza, el ingreso en el tiempo, la tasa de interés, factores demográficos u otras afectaciones): el Enfoque Keynesiano, la Hipótesis del Ingreso Permanente y la Hipótesis del Ciclo de Vida.

La función keynesiana de consumo predice que el consumo presente está fuertemente relacionado con el ingreso presente, la propensión marginal a consumir es menor a la unidad por lo que el porcentaje de ingreso ahorrado aumenta con el ingreso (Villagómez, 1993). Sin embargo, de acuerdo con el trabajo de Smyth (1993), resaltan los hallazgos de Simon Kuznets y Raymond Goldsmith, en donde la relación consumo-ingreso (y por lo tanto el ahorro) de corto y largo plazo no cumplen con la propuesta keynesiana: a corto plazo se confirma la propensión media al consumo decreciente, pero a largo plazo, la propensión media al consumo permanecía constante (inclusive si el ingreso aumentaba) además que el consumo-ahorro de los individuos mostraba no estar relacionado solo con el ingreso del presente periodo.

La Hipótesis del Ingreso Permanente (PIH) y la Hipótesis del Ciclo de Vida (LCH) forman parte de las teorías que surgen como respuesta a la desviación entre lo observado en los estudios empíricos y a lo manifestado en las teorías precedentes sobre el comportamiento del consumo-ahorro.

La PIH fue desarrollada por Milton Friedman (1957), bajo la inquietud de que el consumo actual no es determinado únicamente por el ingreso actual debido a que los individuos tienen una preferencia por una trayectoria de consumo suavizada. En su caso considera más relevante el análisis del ingreso permanente además del efecto del ingreso transitorio, considerando que el individuo ajusta el consumo a sus expectativas de ingreso a largo plazo. En este modelo se considera que tanto ingreso como consumo tienen un componente permanente y otro transitorio (factores de oportunidad o accidentales), sostiene que los individuos consumen una fracción de su ingreso permanente en cada periodo, y la propensión promedio a consumir es igual a la propensión marginal a consumir. Concluye que el ahorro refleja en gran medida los efectos del ingreso transitorio debido a que los individuos prefieren mantener un consumo estable, por lo que este cambio transitorio en el ingreso formará parte del ahorro viendo a éste como un fondo de emergencia. En este sentido, el cambio en el consumo estará más relacionado con los cambios en el ingreso permanente. Dado que pedir prestado es usualmente más sencillo cuando se poseen acti- 
REMEF (The Mexican Journal of Economics and Finance) Desarrollo de un modelo logit para examinar el comportamiento del ahorro en la región 60 centro de México, de acuerdo al perfil de los hogares

vos físicos que aquellos que dependan de las habilidades y capacidades del individuo, la necesidad de ahorrar como una reserva para emergencias se espera sea mayor entre más pequeña sea la riqueza, en proporción, proveniente de estas fuentes "no humanas" (activos físicos o sujetos a una tasa de interés).

La LCH fue desarrollada por Franco Modigliani, estudia el consumo considerando el ciclo de vida del ingreso y las necesidades de consumo de los hogares. Dicha teoría sostiene que el ingreso es bajo al inicio y final de la vida del individuo, acompañado de una baja productividad, en contraste, la etapa media de vida se distingue por un ingreso elevado y una mayor productividad, por lo que el consumo-ahorro dependerá de la distribución de edad de la población. De acuerdo a la revisión elaborada por Modigliani y Ando (1963) en este modelo se considera que el individuo maximiza su función de utilidad que considera el consumo planeado hasta la edad en la que el individuo morirá y sujeto a los recursos que tiene disponibles; dichos recursos están conformados por el valor presente de la suma de la riqueza disponible, el ingreso del periodo actual y el ingreso futuro. Como resultado del proceso de maximización el consumo presente será una función del valor de los recursos del individuo y de una proporción que dependerá de la edad del individuo. Concluye que los recursos que un individuo asigna al consumo (a una edad determinada) dependerán de sus recursos de por vida y no solamente sobre los ingresos actuales. Considerando que el individuo elegirá consumir a una tasa estable (cercana a su consumo de por vida promedio anticipado) es posible apreciar que la magnitud del ahorro en periodos cortos de tiempo será influido por el grado en que los ingresos corrientes se separan de los ingresos promedio de por vida (Modigliani, 1986). Un cambio en el ingreso actual tendrá un efecto sobre la riqueza, pero limitado en el consumo actual, la influencia en el consumo podrá verse reflejada en periodos posteriores de su vida.

En distintos estudios empíricos y experimentales se han encontrado situaciones en las que el comportamiento de los individuos no sigue las directrices descritas por la PIH y LCH, tal es el caso de los trabajos de Souleles (1999), Gapinski (1993) y Smyth (1993) en los cuales se resalta la sensibilidad del ahorro a variables distintas al ingreso o las limitantes de los supuestos restrictivos de las teorías tradicionales.

No solo no se ha llegado a un acuerdo sobre el modelo que debe usarse para estudiar al ahorro, sino que los modelos desarrollados han fallado en proporcionar una explicación adecuada sobre la conducta de ahorro de los hogares (Smyth, 1993), lo que pone de manifiesto la relevancia de buscar enfoques alternativos para el análisis de esta temática. En su investigación, Borsch-Supan y Lusardi (2003) reconocen que la teoría acerca del ahorro ha enfrentado grandes desafíos al compararla con los datos empíricos por lo que se hace necesario el uso de micro datos para entender el comportamiento de ahorro de los hogares.

La Economía Conductual surge como respuesta a resultados observables que contradicen la idea de una expectativa racional y la apreciación de anomalías en el comportamiento de los mercados. Como campo de estudio pretende dar respuesta al comportamiento complejo y cambiante de los individuos, específicamente cuando se trata de estudiar las decisiones de ahorro su fortaleza radica en identificar determinantes y motivadores de dicha conducta.

A pesar de que las personas han sido informadas sobre lo recomendable que es mantener un nivel de ahorro, se ha encontrado que no siempre actúan en su propio beneficio, e incluso se ha encontrado que se presenta una desconexión entre las intenciones y el comportamiento. ¿Por qué las decisiones de ahorro de los individuos no son óptimas a pesar de estar bien intencionadas?, ¿por qué personas que se retiran declaran que de poder regresar el tiempo habrían ahorrado más para la vejez?, estas son algunas de las preguntas que la economía conductual puede responder (Knoll, 2010).

Específicamente la Teoría Prospectiva es utilizada en el análisis del proceso de decisión de los individuos en situaciones de riesgo, considera que las personas evalúan las pérdidas 
Revista Mexicana de Economía y Finanzas Nueva Época, Vol. 14 No. 1, (2019), pp. 57-77 DOI: http://dx.doi.org/10.21919/remef.v14i1.359

y ganancias de una forma diferente a como lo hace la teoría de la utilidad esperada, pues permite considerar individuos que no poseen racionalidad ilimitada, ni autocontrol o que no sean ilimitadamente egoístas.

La Teoría Prospectiva difiere de las consideraciones y supuestos que involucra la LCH y PIH especialmente por las siguientes consideraciones: 1) el individuo se preocupa más de las posibles pérdidas que de las posibles ganancias considerando un punto de referencia, 2) resalta la importancia de cómo los individuos ven una situación determinada antes de tomar una decisión, considerando que los individuos no siempre consideran todas las consecuencias de las decisiones que toman.

La principal aportación de la Teoría Prospectiva al análisis del comportamiento de ahorro de los hogares es permitir la incorporación de la aversión a la pérdida al modelo de análisis, este término es relevante en el sentido que permite conocer a mayor detalle el modo en que los individuos toman decisiones. El conocimiento adicional puede ser utilizado como material en el diseño de estrategias de promoción del ahorro, captación de recursos o diseño de planes de inversión.

El desarrollo y postulados de la Teoría Prospectiva se basan en el estudio de los procesos cognitivos y los sesgos (biases) a los que se enfrenta un individuo al tomar una decisión, en este sentido, la incorporación de la Teoría Prospectiva al estudio de un fenómeno económico debe tomar en cuenta, como ocurren los sesgos en el contexto del fenómeno utilizado, como se manifestarían y como podrían detectarse.

Las principales críticas a la Teoría Prospectiva (Andersen Nowak, 2013) se centran en que el comportamiento observado en estudios experimentales no es aplicable al análisis de situaciones de mercado dado que el aprendizaje y la competencia implicaría un comportamiento aproximativo a la racionalidad. Dado lo anterior, las diferencias observables entre las decisiones que toman los individuos y los modelos tradicionales podrían ser tratadas como desviaciones que se eliminarían mediante un análisis con un mayor nivel de agregación, o en cambio, podría ser un indicio de que los modelos tradicionales no son adecuados para explicar el comportamiento humano. Es así que la principal limitante de la Teoría Prospectiva se centra en la pertinencia para estudiar el fenómeno económico específico que se pretende analizar, así como realizar las adecuaciones necesarias al modelo utilizado. Un ejemplo de la dificultad para su implementación y adaptación de sus principios a diversos modelos es resaltada en el trabajo de Castro y Parsons (2014), específicamente para el caso de la modelación basada en agentes.

De forma específica, múltiples investigaciones han encontrado en la Teoría Prospectiva un marco de referencia para el estudio del ahorro, presentando hallazgos que indican la pertinencia de su incorporación al estudio de esta temática (Fisher Montalto, 2011).

El modelo de decisiones de consumo y ahorro propuesto por Botond Koszegi y Matthew Rabin (2006), que toma en consideración la directriz de la Teoría Prospectiva para incorporar la aversión a la pérdida, define que el ahorro precautorio es motivado por la incertidumbre en el ingreso como una medida para disminuir el malestar que llegaría ante una disminución en el consumo; los individuos tienen una tendencia a sobre consumir porque tienen un incentivo para sorprenderse a sí mismos con consumo adicional. Estas dos ideas llevan a que el consumo a lo largo de la vida tenga una forma cóncava, en la cual el individuo prefiere un menor consumo futuro que disminuir el consumo actual. Cuando en ese tiempo futuro el consumo llegue efectivamente a ser menor, el malestar será limitado porque para ese entonces las expectativas se habrán ajustado a la baja.

Adicionalmente, en el trabajo de Fredrickson (2013) se examina la asimetría en la respuesta de los hogares ante ganancias o pérdidas en el nivel de ingreso actual, el ingreso anticipado y el valor de los activos, además de estudiar la forma en la que dicha asimetría en la respuesta influye en el ahorro. Encuentra que la Teoría Prospectiva y otras variables conductuales contribuyen a predecir el comportamiento de ahorro de los hogares, confirma que los hogares tienen una respuesta más acentuada ante la caída de las variables de 
REMEF (The Mexican Journal of Economics and Finance)

Desarrollo de un modelo logit para examinar el comportamiento del ahorro en la región centro de México, de acuerdo al perfil de los hogares

ingreso que ante un aumento de las mismas, además de que el aumento en la incertidumbre en el ingreso disminuye las posibilidades de ahorro.

Dentro de las investigaciones desarrolladas para el estudio del ahorro en México los trabajos de Miguel Székely (1998) y Alejandro Villagómez (2008) son referentes para cualquier investigación que hable del ahorro en el país; el primero estudia el nivel de ahorro de los hogares, los instrumentos financieros que se utilizan para ahorrar y las características de los hogares que ahorran encontrando que la ocupación y la posición de empleo son relevantes al momento de determinar el nivel de ahorro, el cual se concentra en los deciles de mayor ingreso; por su parte, Villagómez hace una revisión de la estructura, evolución y determinación del ahorro desde 1960, en el estudio de la tasa de ahorro encuentra que ésta crece con la edad hasta alcanzar un máximo a la edad de 55 años y decreciendo conforme se acerca la edad de retiro, este comportamiento coincide con el movimiento del perfil de ingreso de los trabajadores.

Entre los trabajos más recientes sobre el ahorro en el país es posible mencionar el artículo de Brown, Dominguez, y Nava (2014) que resalta las diferencias en los factores que influyen en el ahorro de los hogares de acuerdo al género del jefe de familia, al respecto confirman que el ahorro se concentra en los deciles más altos y que la respuesta ante variables como los apoyos gubernamentales, variables institucionales o la escolaridad es diferente de acuerdo al género del jefe de familia. En el documento de Aguilar y Valles (2015) se estudia la relación entre el ahorro y el ingreso, el estatus de trabajo, el acceso a servicios de salud y se incorporan variables relacionadas con el acceso a servicios financieros, el tipo de localidad, edad del jefe de familia y presencia de dependientes económicos; en lo referente al acceso de servicios financieros, que representa la novedad de dicha investigación, se encontró que no tiene una relación significativa con el ahorro, mientras que la posesión de tarjeta de crédito por algún miembro del hogar influye de forma positiva en el ahorro.

Finalmente, el trabajo de (Ceballos, 2018) modela el ahorro de los hogares a lo largo de su ciclo de vida, encontrando que al inicio de la vida familiar se depende en una mayor proporción del crédito, mientras que, al final de la vida se presenta un ahorro elevado y sostenido, producto de una caída en el consumo y que es impulsado por el componente monetario, dicho comportamiento es distinto a lo sostenido por la LCH.

\section{Metodología de análisis}

A efecto de estudiar el comportamiento de ahorro de los hogares se han seleccionado variables que permiten caracterizar perfiles de hogar fácilmente distinguibles, este acercamiento responde a la necesidad de reconocer las necesidades de consumo y ahorro de acuerdo a las distintas etapas de los hogares. Dicho acercamiento permite llevar el análisis a aspectos más allá del estudio del ingreso (Lee, Montalto, Park, 2000).

Existen diversas metodologías para la medición del ahorro haciendo uso de la información recabada en las encuestas oficiales, los diversos enfoques propuestos buscan soslayar la dificultad de trabajar con una fuente de información acerca de los ingresos y gastos declarados, en este sentido es relevante definir un marco de referencia consistente con otras investigaciones en México que cuantifique de manera adecuada el ahorro de los hogares.

A continuación, se describen a detalle las variables utilizadas en la investigación, la definición de ahorro que se seguirá, así como la descripción de los perfiles de hogar propuestos. Todo ello como preámbulo explicativo del modelo logit empleado para estimar la probabilidad de ahorro de los hogares.

\subsection{Descripción de Variables}

Las variables utilizadas para el diseño de los perfiles permiten clasificar a la población en grupos fácilmente distinguibles considerando atributos que han resultado significativos para el estudio del ahorro en otras investigaciones: edad, estado civil, escolaridad, género 
y presencia de menores. Al respecto se ha encontrado en el caso de la edad, que la tasa de ahorro es alta y estable hasta los 45-49, sin embargo, no se presenta un efecto de "desahorro" conforme aumenta la edad del jefe del hogar (Borsch-Supan A. , 2003), este hallazgo justifica la aplicación con cautela de la LCH. El estado civil influye de forma negativa en el ahorro cuando se habla de personas solteras (con o sin hijos), los casados presentan un mayor ahorro (Grinstein-Weiss, Sherraden, Zhan, 2004), especialmente cuando aumenta el riesgo de un divorcio (por ejemplo, cuando se facilitan los trámites para realizarlo) (Gonzalez Ozcan, 2008). Se ha encontrado que el ahorro aumenta con la escolaridad (Grinstein-Weiss Zhan, 2005), y que en aquellos hogares con una alta escolaridad el ahorro aumenta ante una expectativa de reducción en el ingreso (Finaly Price, 2014). En lo referente al género se ha encontrado que cuando aumenta el ingreso y poder de negociación de las mujeres el ahorro agregado también aumenta, lo cual podría sugerir que su propensión a ahorrar es mayor. Finalmente, la presencia de menores dependientes representa un elemento que disminuye el ahorro del hogar (Lee, Montalto, Park, 2000).

La incorporación de variables de incertidumbre en el ingreso permite incorporar a la investigación el estudio de factores que vulneren la conducta de ahorro de los hogares, en otras investigaciones se ha identificado que el estado de salud, el autoempleo y tener solo un ingreso en el hogar tienen un impacto negativo en el ahorro de los hogares (Kennickell Lusardi, 2003) (Copaciu, Croitoru, Mihaescu, 2010) (Skinner, 1988) (Bosworth, Burtless, Sabelhaus, 1991). En el caso del desempleo se sostiene que en realidad el mayor riesgo de sufrirlo aumentaría el ahorro considerando un individuo racional que prevé (Engen Gruber, 2001), se reconoce que el subempleo es una situación en aumento en México (Ruiz Ordaz, 2011) lo cual constituye una condición de precarización laboral. Para la presente investigación se propone considerar la relación que sugiere la Teoría Prospectiva acerca del efecto de la incertidumbre en el ingreso sobre el ahorro, en el sentido de que un aumento en la presencia de dichos factores llevará a una disminución en el ahorro producto de que los hogares tienen aversión a la pérdida del nivel de consumo (Fredrickson, 2013).

El nivel de ingreso del hogar es una variable básica para el estudio del ahorro de los hogares, en diversos estudios se ha analizado a detalle la relación que guarda el ingreso con el ahorro (visto desde distintos enfoques desde el ingreso actual, las fuentes de ingreso, el ingreso permanente o respuesta al ingreso futuro). En el trabajo de Thaler (1990) se muestra que el nivel de ingreso actual influye en su nivel de consumo y por lo tanto de ahorro.

La incorporación de la variable propiedad de una vivienda surge como respuesta de su importancia como destino de recursos de los individuos y la necesidad de ahorro previo antes de su adquisición. Se ha identificado que la propiedad de una vivienda modifica de por vida el comportamiento de ahorro del hogar así como su riqueza futura (Dietz Haurin, 2003).

\subsection{Metodología para la medición del ahorro}

Existen múltiples enfoques para medir el ahorro de los hogares, considerando información de encuestas o de agregados monetarios. Para la presente investigación se considera como punto de partida la definición propuesta por Székely (1998) que considera el ingreso y gasto corrientes de los hogares:

$$
\begin{gathered}
S_{1}=y-c \\
S_{2}=y-c_{n d}
\end{gathered}
$$

Donde:

$S_{1}$ : ahorro corriente

$S_{2}$ : ahorro $S_{1}$ excluyendo gasto en bienes duraderos

$y$ : ingreso corriente 
REMEF (The Mexican Journal of Economics and Finance)

$c:$ gasto corriente

$c_{n d}:$ gasto corriente en bienes no duraderos

Es importante señalar que al concepto de gasto corriente debe incluir el pago que realiza el hogar por concepto de intereses para tener una medida más precisa del ahorro del hogar. Para consultar el cálculo por concepto de intereses que fueron incorporados al gasto corriente revisar el ANEXO 1, para identificar los conceptos de gasto corriente considerados como gasto en bienes duraderos se recomienda consultar a los autores para la lista detallada.

Para el objetivo de la presente investigación se utilizará como variable de ahorro la variable $S_{2}$, que será utilizada para identificar a los hogares con un recurso corriente positivo que les permitiría ahorrar, dicha determinación permite identificar como ahorradores a aquellos hogares que realizan gastos en bienes duraderos, considerados como tal a los gastos que constituyen una inversión que permite a los hogares transferir consumo al futuro.

\subsection{Diseño del perfil del hogar}

El uso del análisis de árboles de decisión permite conformar los distintos perfiles del hogar de acuerdo a las características del jefe del hogar considerando las variables edad, estado civil, escolaridad, género y presencia de menores dependientes. Esta técnica de análisis ha sido utilizada en múltiples trabajos de investigación económica y forma parte de las herramientas de análisis de datos para finanzas (Kovalerchuk Vityaev, 2000).

Los perfiles considerados en la presente investigación coinciden con la clasificación propuesta en (Cruz, 2017), para su elaboración se consideró el uso del algoritmo CHAID (Chi-square Automatic Interaction Detector) para permitir la clasificación de grupos no solo como árbol binario; además se prefirió sobre el algoritmo CHAID extendido para tener muestra suficiente para un análisis regional que permita caracterizar el comportamiento de ahorro de los hogares. El algoritmo de clasificación elegido compara cada una de las variables independientes, eligiendo para cada nivel de clasificación aquellas que presentan interacción más fuerte con la variable dependiente y desagregando aquellas variables que presentan un comportamiento significativamente distinto mediante el estadístico chi-cuadrado. De forma general, la prueba de significancia chi cuadrada considera el cálculo de los cuadrados de las desviaciones de las cantidades observadas respecto a los valores esperados, ponderados por los recíprocos de sus valores esperados, por lo cual tenemos el siguiente estadístico de prueba (Mendenhall, Scheaffer, Wackerly, 2010):

$$
X^{2}=\sum_{i=1}^{k} \frac{\left[n_{i}-E\left(n_{i}\right)\right]^{2}}{E\left(n_{i}\right)}=\sum_{i=1}^{n} \frac{\left[n_{i}-n p_{i}\right]^{2}}{n p I}
$$

Donde:

n:cantidad de elementos total

$n_{i}$ :cantidad de casos en el grupo i

k:número total de grupos

$p_{i}$ :probabilidad de pertenecer al grupo i

Las variables sociodemográficas consideradas para el análisis de árbol de decisión se presentan en la tabla a continuación, considerando la definición de jefe de familia bajo el enfoque de jure (jefatura declarada). 
Cuadro 1. Variables conformación de perfiles

\begin{tabular}{|l|l|}
\hline \multicolumn{1}{|c|}{ VARIABLE } & \multicolumn{1}{c|}{ DESCRIPCIÓN } \\
\hline $\begin{array}{l}\text { Edad del jefe } \\
\text { del hogar }\end{array}$ & Grupos quinquenales de edad. \\
\hline Escolaridad & $\begin{array}{l}\text { Se agrupa el nivel escolar trunco con el terminado, se } \\
\text { considera que las personas con preescolar no tienen } \\
\text { instrucción. }\end{array}$ \\
\hline Género & Género del jefe del hogar declarado. \\
\hline $\begin{array}{l}\text { Presencia de } \\
\text { menores }\end{array}$ & $\begin{array}{l}\text { Presencia en el hogar de integrantes menores de 12 } \\
\text { años. }\end{array}$ \\
\hline $\begin{array}{l}\text { Estado civil del jefe } \\
\text { del hogar }\end{array}$ & Soltero, casado, unión libre, separado/divorciado/viudo. \\
\hline \multicolumn{2}{|c|}{ Fuente: Elaboración propia con información de ENIGH 2014. } \\
\hline
\end{tabular}

Haciendo uso del algoritmo de clasificación CHAID se obtuvieron 12 nodos terminales, la valoración de la precisión del árbol de decisión se realizó mediante una validación cruzada con diez sub muestras, encontrando que la precisión predictiva del árbol es similar al construido con la validación cruzada. La edad del jefe resulta la variable que distingue en mayor medida el comportamiento de ahorro de los hogares, considerando tres grandes grupos: personas menores de 45 años, 45 a 49 años y personas de 50 en adelante. La segunda variable explicativa depende del rango de edad del que se trate, siendo el estado civil lo que diferencia al primer grupo de edad, la escolaridad al segundo grupo y la presencia de menores en el tercero. En algunos de los nodos se llega a un tercer nivel de clasificación, sin embargo, dado que se pretende tener grupos con muestra suficiente para el análisis regional se procedió a agrupar nodos terminales del tercer nivel y prescindir de algunas de las separaciones, es así como se determinaron los perfiles para el estudio del ahorro de los hogares.

Cuadro 2. Perfil del hogar de acuerdo al nivel de ahorro

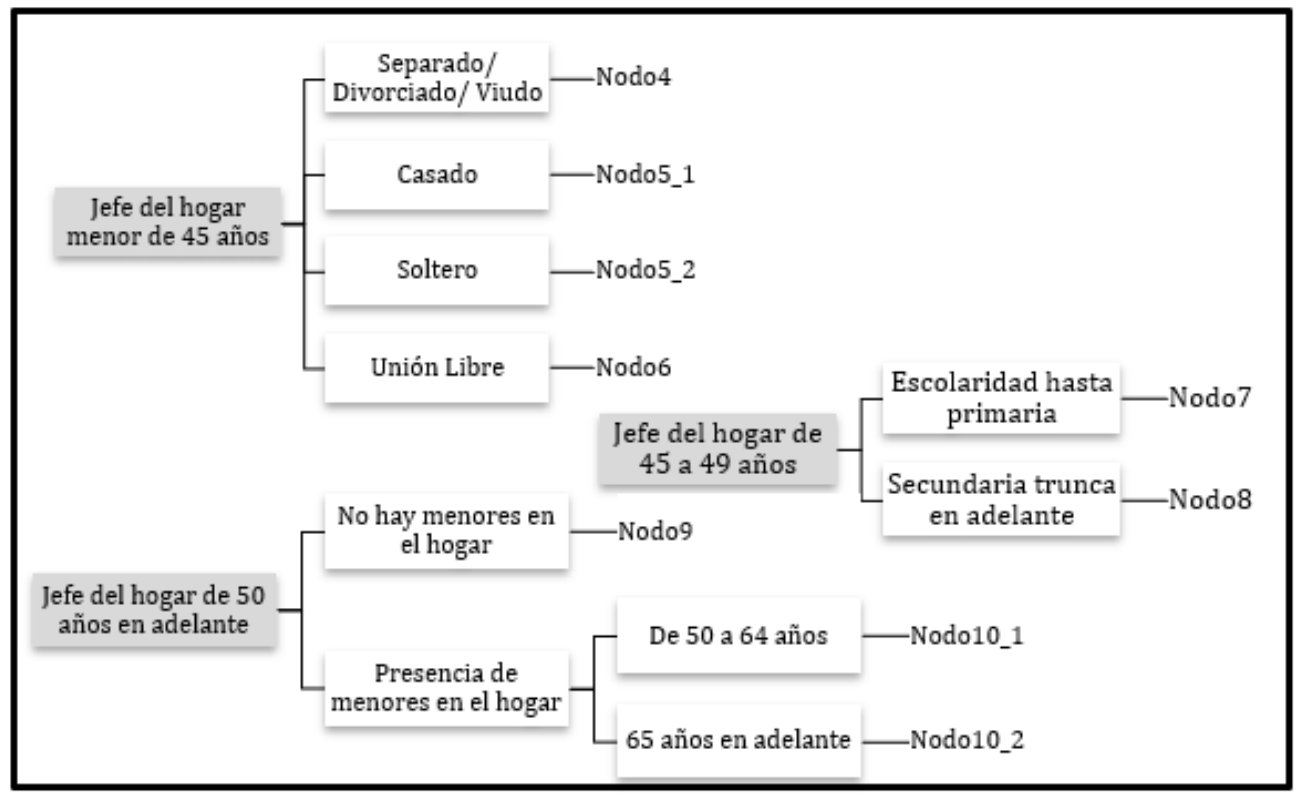

Fuente: Elaboración propia con información de ENIGH 2014

Es importante señalar que, a pesar de incluir el género del jefe del hogar dentro de las cinco variables a considerar para la determinación de los perfiles, se encontró que otras variables como la edad, estado civil, escolaridad o presencia de menores dependientes 
REMEF (The Mexican Journal of Economics and Finance)

permitían identificar una mayor diferenciación entre los grupos, por lo cual se prescindirá de la distinción por género para la presente investigación.

\subsection{Estadística descriptiva perfiles del hogar}

En esta sección se revisarán los hallazgos principales de la incidencia de las variables de interés para cada uno de los perfiles en la región centro, el cuadro detallado puede consultarse en el ANEXO 2.

\subsubsection{Nivel de ingreso}

En el trabajo de Villagómez (2008) se identifica que el perfil de ingreso de los individuos aumenta con la edad alcanzando un máximo alrededor de los 50 años. Al analizar el nivel de ingreso corriente total por hogar para cada uno de los perfiles se aprecia, en la Gráfica 1, que al aumentar la edad del jefe de familia el ingreso por hogar no disminuye. Sin embargo, si se considera un nivel de ingreso normalizado por el número de perceptores de ingreso corriente monetario del hogar (ecuación 4), se aprecia que en realidad el mayor ingreso por hogar en aquellos con jefe de familia mayor de 49 años viene acompañado por un mayor número de perceptores de ingreso.

$$
I N=\frac{y}{P}
$$

Donde:

IN:Ingreso corriente normalizado

$y$ : Ingreso corriente

P:Perceptores de ingreso corriente monetario del hogar

Para el rango de edad de menores de 45 años, el ingreso es mayor en hogares de solteros y casados, los primeros con el ingreso por hogar normalizado más alto de todos los grupos. En el caso del grupo de 45 a 49 años se aprecia que cuando el jefe del hogar alcanza un nivel de estudios de por lo menos secundaria el ingreso del hogar tiene una mediana significativamente mayor que en aquellos hogares con un jefe de menor escolaridad.

A nivel de ingreso corriente total, los hogares con presencia de menores de 12 años, muestran un mayor ingreso; al observar el ingreso normalizado se aprecia que en realidad el nivel de ingreso por hogar es menor con el aumento de la edad del jefe de familia y en aquellos hogares con menores de 12 años. A pesar del bajo nivel de ingreso normalizado de estos hogares se considera que tienen potencial de ahorro ya que al considerar los ingresos combinados de todos los perceptores el hogar en su conjunto resultan ser el perfil de hogar con mayor ingreso.

Gráfica 1. Ingreso corriente por perfil del hogar

Mediana de ingreso (Pesos trimestrales)

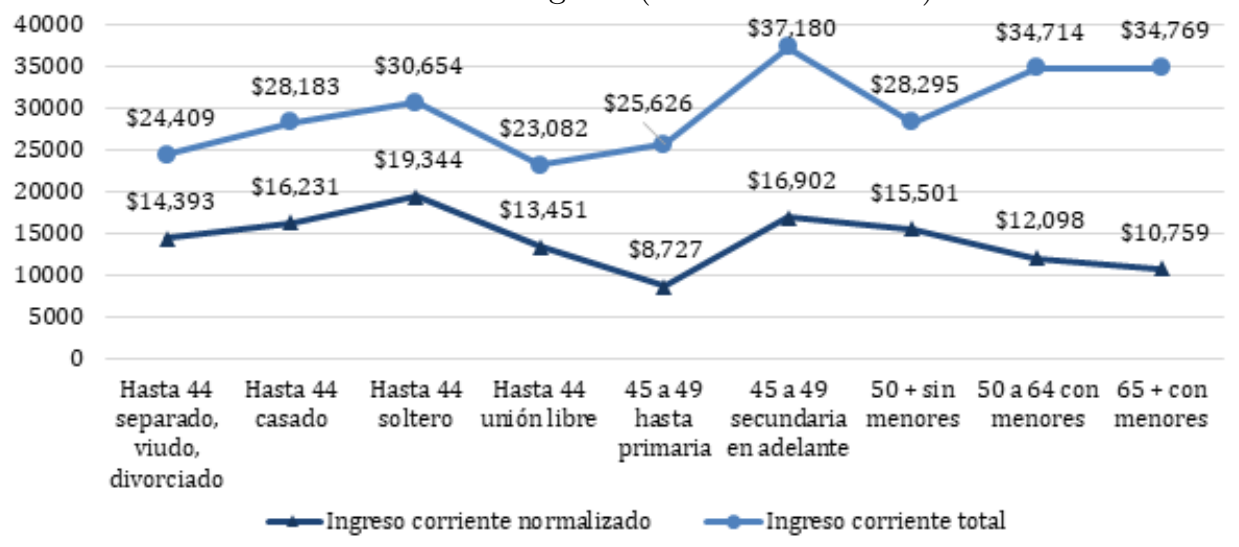

Fuente: Elaboración propia con información de ENIGH 2014 


\subsubsection{Propiedad de una vivienda}

La incidencia de vivienda en proceso de pago es mayor en el grupo de edad de menores de 45 años, especialmente en el perfil de personas casadas (16.2\%), estos hogares se encuentran en un momento de construcción del patrimonio. La mayor parte de los solteros (el grupo de mayor ingreso) no habitan en una vivienda de su propiedad.

Sobresale en el grupo de 45 a 49 años con escolaridad de secundaria en adelante que el $17.6 \%$ continúan pagando su vivienda, lo que contrasta con aquellos de menor escolaridad en el mismo grupo de edad donde solo el $5.1 \%$ continúa en esta situación.

En los hogares con jefe de 50 años de edad en adelante, la mayor parte posee una vivienda propia, especialmente en el caso de los mayores de 64 años y presencia de menores de 12 años en el hogar. El habitar una vivienda pagada permite tener una mayor cantidad de recursos disponibles como hogar dado que no tienen un crédito hipotecario ni se enfrentan al pago de una renta.

\subsubsection{Variables socioeconómicas}

De acuerdo al INEGI (2017) se identifican 5 tipos de hogares en México de acuerdo a la estructura de parentesco de los integrantes del hogar, de acuerdo al cuadro a continuación.

Cuadro 3. Tipo de hogar

\begin{tabular}{|l|l|}
\hline TIPO DE HOGAR & \multicolumn{1}{c|}{ DESCRIPCIÓN } \\
\hline Nucleares & Parejas con/sin hijos. \\
\hline Ampliados & Hogar nuclear y otros parientes. \\
\hline Compuestos & $\begin{array}{l}\text { Nuclear o ampliado y personas sin } \\
\text { parentesco con el jefe del hogar. }\end{array}$ \\
\hline Unipersonales & Solo una persona en el hogar. \\
\hline Corresidentes & $\begin{array}{l}\text { Dos o más personas sin relaciones de } \\
\text { parentesco. }\end{array}$ \\
\hline
\end{tabular}

Fuente: Elaboración propia con información de INEGI 2017.

En los hogares compuestos por diversos núcleos familiares es posible identificar situaciones en las que la fuente de ingreso pueda provenir de múltiples perceptores de ingreso y con ello influir en la probabilidad de ahorro.

En el grupo de edad menor de 45 años, la mayor parte de los hogares son nucleares (68.2\% a $88.1 \%$ ), a excepción del grupo de solteros que son en su mayoría unipersonales $(43.1 \%)$. La segunda incidencia en este grupo de edad son los hogares ampliados y compuestos en los que se comparte vivienda con otros parientes o personas sin parentesco. En los hogares con jefe de 45 a 49 años se repite la mayor presencia de hogares nucleares, $(66.6 \%$ a $70.0 \%)$ pero aumenta la proporción de aquellos ampliados y compuestos (especialmente en el grupo de menor ingreso). Para los hogares con jefe de 50 años en adelante se identifica que la presencia de menores de 12 años en el hogar está relacionada con una mayor proporción de hogares ampliados y compuestos por lo que se distingue que los menores no son hijos directos del jefe de familia sino de otras personas que viven en el hogar.

Para identificar el comportamiento del resto de variables (edad, género, escolaridad, presencia de menores, estado civil) en cada uno de los perfiles se construyó una tabla cruzada; dado que estas variables fueron utilizadas para construir en sí mismo los perfiles solo se resaltan los hallazgos principales del cruce con el objetivo de conocer a mayor detalle las características del jefe del hogar.

En lo referente a la edad, sobresale que los jefes del hogar solteros o en unión libre reportan la menor edad promedio de su grupo, además que la escolaridad entre los jefes de 45 a 49 años no modifica su edad promedio.

Para el grupo menor de 45 años en donde el jefe de familia está casado o en unión 
REMEF (The Mexican Journal of Economics and Finance)

Desarrollo de un modelo logit para examinar el comportamiento del ahorro en la región

libre se identifica al hombre como el jefe del hogar, en aquellos de personas separadas/divorciadas/viudas se identifica a una mujer en este rol, en el caso del grupo de solteros hay una mayor proporción de mujeres $(54.7 \%)$. En el resto de los grupos de edad en la mayoría de los hogares se identifica a un hombre como el jefe del hogar, sin embargo, en aquellos sin presencia de menores, con menores y jefe mayor de 64 años, la presencia de hombres es ligeramente menor que en otros grupos, posiblemente debido a la mayor edad de supervivencia de las mujeres.

En cuanto a la escolaridad, para el grupo menor de 45 años, la menor escolaridad se presenta en los separados/divorciados/viudos y en unión libre, quienes alcanzan mayor grado escolar son quienes permanecen solteros; en el grupo de edad de 50 en adelante se presenta una menor escolaridad del jefe de familia comparado con el resto de grupos de edad.

Respecto a la presencia de menores, en el grupo menor de 45 años hay mayor presencia de menores entre aquellos que tienen una pareja (78.5\% a $81.0 \%$ ); en los hogares de 45 a 49 aumenta ligeramente la incidencia cuando el jefe tiene una escolaridad menor $(53.1 \%)$ (recordemos que una mayor proporción de estos hogares son ampliados /compuestos).

Por lo que refiere al estado civil, la mayor parte de los hogares de 45 años en adelante son representados por un jefe del hogar casado $(52.8 \%$ a $63.7 \%)$, la presencia de jefes de familia viudos aumenta a partir de los 65 años (33.6\%).

\subsubsection{Factores de incertidumbre}

Ahora bien, en relación con la incidencia de los factores de incertidumbre de acuerdo al perfil del hogar se encontró que la presencia de un problema de salud que impidiera al jefe del hogar atender sus actividades y solicitara atención médica es mayor en el grupo de personas menores de 45 años y separadas/solteras/viudas y en el grupo de 50 años en adelante.

Con respecto al autoempleo, esta situación de empleo se presenta con mayor frecuencia en el grupo de 45 a 49 años, especialmente en aquellos con menor escolaridad. Para el grupo de menores de 45 años la menor incidencia es en aquellos solteros (que son también los de mayor escolaridad). Para el jefe del hogar de 50 años en adelante, el autoempleo, continua como como una opción laboral para el $23.1 \%$ al $27.3 \%$ de los hogares.

En lo que respecta al nivel de ocupación del jefe del hogar se encontró que los jefes del hogar de 45 a 49 años con escolaridad de secundaria en adelante representan el perfil con mayor presencia de desempleo reciente del jefe del hogar $(4.1 \%)$. Además, en los hogares del menor grupo de edad la presencia de subempleo del jefe del hogar es menor en aquellos casados o en unión libre. Para el grupo de 45 a 49 años el subempleo se acentúa en quienes tienen una escolaridad menor. Para el grupo de 50 años en adelante la presencia de subempleo es similar entre los perfiles (14.6\% a $15.0 \%$ ).

Finalmente, en lo que refiere al número de personas ocupadas, se encontró que en el grupo de menor edad los hogares de personas solteras son quienes tienen una mayor proporción de integrantes que se encuentran ocupados (perciben un ingreso monetario y tienen trabajo). En aquellos de 50 años en adelante con presencia de menores de 12 años se identifica que la proporción de integrantes ocupados disminuye, debido en parte a la presencia de dependientes económicos.

\section{Modelo de probabilidad de ahorro de los hogares}

En la gráfica 2 se presenta el nivel de ahorro de los hogares de acuerdo a la definición S2 (ahorro excluyendo el gasto en bienes duraderos) considerando el perfil de cada uno de éstos. Si se analiza la proporción de hogares con el potencial de ahorrar (aquellos con una diferencia positiva de acuerdo a la definición $S_{2}$ ), se aprecia que dicha proporción aumenta con la edad del jefe del hogar, siendo el grupo de 50 años en adelante el que contiene un mayor número de hogares con una diferencia positiva. 
La razón de ahorro definida como el nivel de ahorro de acuerdo al ingreso corriente del hogar es presentada en la gráfica 2 en conjunto con la razón de ahorro normalizada para identificar el nivel posible de ahorro de cada uno de los perfiles. Se considera para su cálculo únicamente a los hogares con una diferencia positiva de acuerdo a la definición de ahorro $S_{2}$.

$$
\begin{aligned}
& R S_{2}=\frac{S_{2}}{y} \\
& R S_{2} N=\frac{S_{2}}{y P}(6)
\end{aligned}
$$

Donde:

$R S_{2}$ : Razón de ahorro $S_{2}$

$R S_{2} N$ : Razón de ahorro $S_{2}$ normalizado

$S_{2}$ : Ahorro $S_{1}$ excluyendo gasto en bienes duraderos

$y$ : Ingreso corriente

$P$ : Perceptores de ingreso corriente monetario del hogar

En el grupo de menores de 45 años hay un mayor número de hogares con potencial de ahorrar entre aquellos casados. Al observar la razón de ahorro normalizado se aprecia que el grupo de separados / viudos / divorciados presentan el nivel más bajo del indicador dentro de su grupo de edad. El grupo de solteros son quienes tienen un nivel más alto de su grupo, sin embargo, la proporción de hogares con una diferencia positiva en $S_{2}$ es una de las más bajas de todos los perfiles, a pesar de distinguirse como un perfil de alto ingreso y escolaridad en su conjunto no se está traduciendo en mayor ahorro.

En los hogares con jefe de 45 a 49 años la proporción de hogares ahorradores es ligeramente mayor en aquellos con menor escolaridad, inclusive su razón de ahorro (normalizada y no normalizada) es mayor que el perfil con mayor escolaridad dentro del rango de edad. Recordemos que quienes tienen escolaridad de secundaria en adelante continúan pagando su vivienda por lo que esta condición puede explicar el nivel menor de sus indicadores de ahorro.

En el grupo de edad de 50 en adelante hay un mayor número de hogares con potencial de ahorrar, su razón de ahorro es alta visto como hogar (20.1\% a 22.5\%), al analizar la razón normalizada se aprecia que en realidad la razón de ahorro por integrante perceptor de ingreso es menor que la de otros grupos, sin embargo, debe seguir resaltando el potencial de ahorro como hogar por el hecho de que una mayor proporción de éstos tiene una diferencia positiva en la definición de ahorro $S_{2}$.

Gráfica 2. Ahorro S2 por perfil del hogar

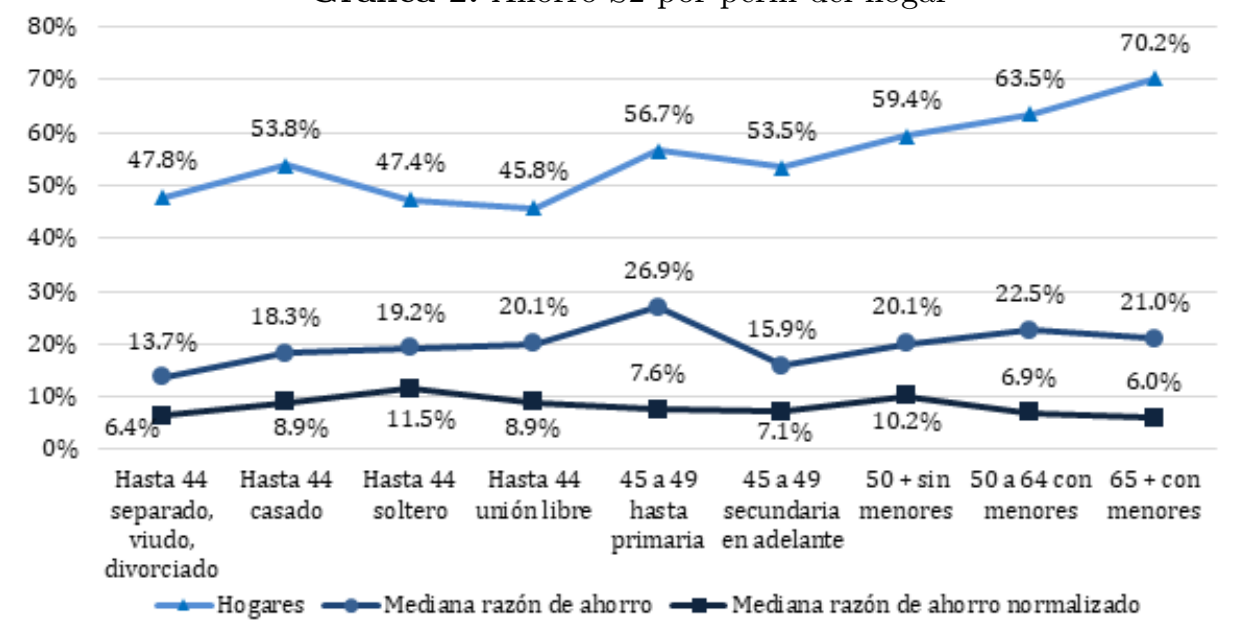

Fuente: Elaboración propia con información de ENIGH 2014 
REMEF (The Mexican Journal of Economics and Finance)

\subsection{Cálculo de la probabilidad de ahorro}

Para el cálculo de la probabilidad de que un hogar ahorre se considera la siguiente clasificación de variables.

Cuadro 4. Descripción de variables VARIABLE DEPENDIENTE

\begin{tabular}{|c|c|}
\hline \multicolumn{2}{|c|}{ VARIABLE DEPENDIENTE } \\
\hline Ahorro del hogar & $\begin{array}{l}\text { Si el hogar presenta un saldo positivo en el } \\
\text { cálculo del nivel de ahorro S2 se considera } \\
\text { que ahorra. }\end{array}$ \\
\hline \multicolumn{2}{|c|}{ VARIABLES EXPLICATIVAS } \\
\hline Perfil al que pertenece el hogar & $\begin{array}{l}\text { La referencia es sobre aquellos hogares del } \\
\text { menor rango de edad y que no tienen pareja } \\
\text { (solteros, divorciados, separados y viudos). } \\
\text { Los perfiles son identificados con el número } \\
\text { de nodo que se muestra en el Cuadro } 2 \text {. }\end{array}$ \\
\hline \multirow{4}{*}{$\begin{array}{l}\text { Situaciones que aumentan la } \\
\text { incertidumbre del ingreso } \\
\text { del hogar }\end{array}$} & $\begin{array}{l}\text { Enfrentar un problema de salud reciente que } \\
\text { requirió de atención médica (problema_salud2) }\end{array}$ \\
\hline & $\begin{array}{l}\text { Ser autoempleado, no tienen un trabajo } \\
\text { subordinado y trabajaron por su cuenta } \\
\text { (autoempleo). }\end{array}$ \\
\hline & $\begin{array}{l}\text { Enfrentó una situación reciente de desempleo } \\
\text { o se encuentra subempleado (ocupacion_d). }\end{array}$ \\
\hline & $\begin{array}{l}\text { Personas ocupadas que reciben un ingreso } \\
\text { monetario en el hogar, se considera la } \\
\text { proporción de personas ocupadas del total } \\
\text { de personas en el hogar (perc_ocupa_norm). }\end{array}$ \\
\hline \multirow[b]{2}{*}{ Variables adicionales } & Ingreso corriente trimestral del hogar (ing_cor) \\
\hline & $\begin{array}{l}\text { Propiedad de una vivienda, ya sea que se esté } \\
\text { pagando (t_vivienda_pagando) o esté pagada } \\
\text { (t_vivienda_pagada). }\end{array}$ \\
\hline
\end{tabular}

Fuente: Elaboración propia de acuerdo a las variables propuestas en (Cruz, 2017), para la presente investigación se incorpora al análisis la variable de subempleo a efecto de estudiar el efecto en el ahorro de la precarización laboral.

La ecuación estimada es la siguiente:

$$
\begin{gathered}
P(\text { ahorro })=B_{0}+B_{1} \text { nodof } 5_{1}+B_{2} \text { nodof } 6+B_{3} \text { nodof } 7+B_{4} \text { nodof } 8+B_{5} \text { nodof } 9+ \\
B_{6} \text { nodof } 10_{1}+B_{7} \text { nodof } 10_{2}+B_{8} \text { problema_salud } 2+B_{9} \text { autoempleo }+ \\
B_{10} \text { ocupacion_d }+B_{11} \text { perc_ocupa_norm }+B_{12} i n g \_c o r+B_{13} t \_v i v i e n d a \_p a g a n d o+ \\
B_{14} t \text { vivienda_pagada }
\end{gathered}
$$

Los resultados de la regresión logística muestran los resultados del modelo calculado para la región centro del país, los perfiles propuestos, variables que influyen en la incertidumbre del ingreso y las variables adicionales propuestas resultaron ser significativas para el problema de estudio. 
Cuadro 5. Resultados regresión logística Región Centro

\begin{tabular}{|l|c|}
\multicolumn{2}{c}{ Coeficientes } \\
\hline Variables explicativas & Nacional \\
\hline nodof5_1 & 0.3941873 \\
\hline nodof6 & 0.3538192 \\
\hline nodof7 & 0.6049629 \\
\hline nodof8 & $0.2364061^{* *}$ \\
\hline nodof9 & 0.5555028 \\
\hline nodof10_1 & 0.7025577 \\
\hline nodof10_2 & 1.102366 \\
\hline problema_salud2 & $-0.1222399^{*}$ \\
\hline autoempleo & -0.3895537 \\
\hline desempleo & -0.1801237 \\
\hline perc_ocupa_norm & 0.7326918 \\
\hline ing_cor & 0.0000166 \\
\hline t_vivienda_pagando & -0.4215897 \\
\hline t_vivienda_pagada & 0.2330915 \\
\hline cons & -1.147655 \\
\hline Pseudo R2 & 0.0627 \\
\hline
\end{tabular}

*Significativo al $95 \%$ de confianza

* Significativo al $90 \%$ de confianza

Fuente: Elaboración propia con información de ENIGH 2014

Se procedió a calcular la probabilidad de ahorro para cada uno de los perfiles cuando no se presentan ninguna de las variables que aumentan la incertidumbre en el ingreso, su vivienda ha sido pagada y tienen un comportamiento promedio en términos de ingreso trimestral $(\$ 37,682.50)$ y personas ocupadas $(46.2 \%$ de los integrantes del hogar).

La probabilidad de ahorro aumenta con la edad del jefe de familia, siendo los hogares con jefe mayor de 65 años y presencia de menores quienes muestran una probabilidad de ahorro mayor.

Cuadro 6. Probabilidad de ahorro de acuerdo al perfil del hogar

\begin{tabular}{|l|c|}
\hline \multicolumn{1}{|c|}{ Perfil } & Probabilidad de ahorro \\
\hline Menor de 45 años sin pareja & $51.24 \%$ \\
\hline Menor de 45 años casado & $60.91 \%$ \\
\hline Menor de 45 años unión libre & $59.95 \%$ \\
\hline 45 a 49 años escolaridad hasta primaria & $65.80 \%$ \\
\hline 45 a 49 años secundaria en adelante & $57.10 \%$ \\
\hline 50 años en adelante sin menores & $64.68 \%$ \\
\hline 50 a 64 años con menores & $67.96 \%$ \\
\hline 65 años en adelante con menores & $75.98 \%$ \\
\hline
\end{tabular}

Fuente: Elaboración propia con información de ENIGH 2014

\subsection{Efecto de los factores de incertidumbre, ingreso y propiedad de una vivienda}

El $55.0 \%$ de los hogares de la región centro están expuestos a por lo menos un factor que aumenta la incertidumbre en el ingreso del tipo autoempleo, desempleo-subempleo o problema de salud. Aquellos hogares en donde el jefe de familia se enfrentó a una situación únicamente de desempleo/subempleo o de problema de salud fueron los que presentaron un menor promedio de personas ocupadas; en el resto de combinaciones el promedio de personas ocupadas es mayor incluso que en aquellos hogares en donde no se presenta ninguno de los factores de incertidumbre por lo que es posible los hogares estén 
REMEF (The Mexican Journal of Economics and Finance)

compensando la situación laboral del jefe del hogar con un mayor número de perceptores de ingreso.

Cuadro 7. Hogares con presencia de factores de incertidumbre

\begin{tabular}{|c|c|c|c|c|}
\hline $\begin{array}{c}\text { Desempleo y } \\
\text { subempleo }\end{array}$ & Autoempleo & $\begin{array}{c}\text { Problema de } \\
\text { Salud }\end{array}$ & $\begin{array}{c}\text { Incidencia } \\
\text { hogares }\end{array}$ & $\begin{array}{c}\text { Promedio personas } \\
\text { ocupadas }\end{array}$ \\
\hline SI & NO & NO & $6.10 \%$ & $47.85 \%$ \\
\hline SI & SI & NO & $3.60 \%$ & $59.24 \%$ \\
\hline SI & NO & SI & $3.30 \%$ & $52.73 \%$ \\
\hline NO & SI & SI & $4.60 \%$ & $51.69 \%$ \\
\hline NO & SI & NO & $11.00 \%$ & $52.41 \%$ \\
\hline NO & NO & SI & $24.40 \%$ & $38.32 \%$ \\
\hline SI & SI & SI & $2.10 \%$ & $63.67 \%$ \\
\hline NO & NO & NO & $45.00 \%$ & $43.38 \%$ \\
\hline
\end{tabular}

Fuente: Elaboración propia con información de ENIGH 2014

De acuerdo a los coeficientes obtenidos como resultado de la regresión logística, el hecho de que el jefe de familia tenga una posición de autoempleo, desempleo/subempleo o sufriera un problema de salud reciente disminuye la probabilidad de ahorro del hogar (mencionados de mayor a menor disminución de la probabilidad de ahorro). El aumento en la proporción de personas ocupadas en el hogar aumenta la probabilidad de ahorro.

Si se calcula la probabilidad de ahorro de un perfil modelo (menores de 45 años sin pareja, ingreso trimestral promedio y número de personas ocupadas promedio, con una vivienda pagada) se obtiene un valor de $51.24 \%$. Para medir los efectos marginales de las variables de incertidumbre se presentan en el cuadro 8 la variación en la probabilidad de ahorro cuando se presenta una a la vez; el autoempleo es la variable con mayor influencia para disminuir la probabilidad de ahorro, ésta disminuye en alrededor del $10 \%$ ante la presencia de dicha situación de empleo. Referente a la proporción de personas ocupadas, un hogar de 4 integrantes tendría que pasar de 2 a 3 ocupados (75\% de integrantes ocupados) para aumentar su probabilidad de ahorro con alrededor de $5.23 \%$ adicionales.

Cuadro 8. Efecto de los factores de incertidumbre

\begin{tabular}{|l|c|}
\hline \multicolumn{1}{|c|}{ Variable } & $\mathbf{d y} / \mathbf{d x}$ \\
\hline Problema de salud & $-3.05 \%$ \\
\hline Autoempleo & $-9.66 \%$ \\
\hline Desempleo o subempleo & $-4.49 \%$ \\
\hline Proporción personas ocupadas & $18.31 \%$ \\
\hline
\end{tabular}

Fuente: Elaboración propia con información de ENIGH 2014

En cuanto a la propiedad de una vivienda, tomando el perfil modelo, estar pagando una vivienda disminuye la probabilidad de ahorro del hogar respecto de aquellos hogares que habitan una vivienda no propia, esta disminución en la probabilidad de ahorro es incluso mayor que el aumento que se presenta en la probabilidad de ahorro al habitar una vivienda propia y pagada.

Cuadro 9. Efecto de la propiedad de una vivienda

\begin{tabular}{|l|c|}
\hline Variable & $\mathbf{d y} / \mathbf{d x}$ \\
\hline Pagando & $-10.43 \%$ \\
\hline Pagada & $5.81 \%$ \\
\hline
\end{tabular}

Fuente: Elaboración propia con información de ENIGH 2014

Un mayor ingreso corriente trimestral dentro del hogar aumenta la probabilidad de ahorro, por ejemplo, si al salario promedio de $\$ 37,682.5$ trimestrales se le aumenta un monto cercano a un salario mínimo trimestral $(\$ 7,203.60)$ aumentaría la probabilidad de ahorro para pasar de $51.24 \%$ a $54.21 \%$. 
Revista Mexicana de Economía y Finanzas Nueva Época, Vol. 14 No. 1, (2019), pp. 57-77 DOI: http://dx.doi.org/10.21919/remef.v14i1.359

Cuadro 10. Efecto del ingreso

Variable $\quad \mathrm{dy} / \mathrm{dx}$

Ingreso trimestral $\quad 4.14 \mathrm{e}-06$

Fuente: Elaboración propia con información de ENIGH 2014

\section{Conclusiones}

Los perfiles del hogar utilizados permiten profundizar en un análisis regional para los estados del centro del país manteniendo la significancia explicativa del modelo nacional de análisis del ahorro de los hogares presentado en el trabajo de (Cruz, 2017). Con esta clasificación se identifican perfiles de ingreso y gasto permitiendo una segmentación de la población más allá de grupos de edad del jefe de familia (a diferencia de la $\mathrm{LCH}$ ).

El ingreso corriente total por hogar es mayor en el grupo de solteros menores de 45 años, jefes con escolaridad de secundaria en delante de 45 a 49 años y jefes de 50 años en adelante con menores en el hogar, sin embargo, al observar las cifras normalizadas se aprecia que en los grupos de edad de 50 años en adelante el ingreso por hogar es en realidad menor al considerar a la cantidad de perceptores de ingreso. Este comportamiento resalta la necesidad de considerar la composición de los hogares como un indicador adicional para identificar el potencial de ahorro como hogar, especialmente para aquellos ampliados y compuestos.

En general, el ingreso resulta una variable que incide de forma positiva en la probabilidad de ahorro, sin embargo, sobresale que los menores de 45 años solteros poseen un mayor ingreso que no se traduce en una mayor proporción de hogares con la posibilidad de ahorrar. De acuerdo con Diamond Vartiainen (2007), se considera como un problema de autocontrol la incapacidad de aceptar un nivel de consumo menor en un periodo a cambio de un nivel de consumo más alto en otro, por lo cual el grupo de solteros menores de 45 años presentan un comportamiento consistente con la falta de autocontrol.

En el grupo de edad de 45 a 49 años se aprecia que aquellos con una menor escolaridad tienen una mayor proporción de hogares con posibilidad de ahorrar y una mediana de razón de ahorro mayor a quienes tienen escolaridad de secundaria en adelante, esto a pesar de ser un perfil con bajo ingreso. Dicho comportamiento requeriría una investigación posterior a efecto de estudiar el efecto de otras variables en dicho grupo, por ejemplo, la influencia de estar en proceso de pago de su vivienda.

Los perfiles de 50 años en adelante se distinguen como los hogares con mayor potencial de ahorro en términos de una mayor probabilidad, a pesar de que su razón de ahorro normalizado no sobrepasa a la de otros perfiles. En términos de implicaciones en políticas públicas es relevante no perder de vista a este sector, puesto que en su mayoría son hogares de corresidencia (en un mismo hogar habita más de un núcleo familiar), lo cual implica que una estrategia diseñada para este tipo de hogares tendría alcance a múltiples perceptores de ingreso.

La incorporación de los factores de incertidumbre en el ingreso contribuyó en el análisis de la conducta de ahorro de los hogares al permitir identificar condiciones de actividad del jefe de familia que disminuyen la probabilidad de ahorro de los hogares. De acuerdo al enfoque de la Teoría Prospectiva, al considerar individuos que tienen aversión a la pérdida (de consumo) que se encuentren en una situación de ingreso incierto, se esperaría que los individuos reduzcan su ahorro antes de reducir su consumo como un mecanismo para evitar sufrir dicha pérdida (Fredrickson, 2013). Los hallazgos de la presente investigación coinciden con lo predicho por la Teoría Prospectiva respecto a las variables que aumentan la incertidumbre en el ingreso, encontrando que aquellos hogares en donde el jefe de familia enfrenta una condición de precariedad laboral (autoempleo, desempleo o subempleo), un problema de salud reciente que requirió atención médica así como un menor número de perceptores de ingreso en el hogar tienen una probabilidad de ahorro menor.

Con ello se confirma la pertinencia de su aplicación a estudios relacionados con la 
REMEF (The Mexican Journal of Economics and Finance)

conducta de ahorro de los hogares, comprobando dos de los elementos principales de la Teoría Prospectiva: la aversión a la pérdida y la respuesta diferenciada ante pérdidas y ganancias (Barberis, 2013). Dicha respuesta se pone de manifiesto en los resultados que arroja la presenta investigación al identificar que los hogares son aversos a la pérdida (de consumo), además de que el efecto de las variables que aumentan la incertidumbre en el ingreso es más acentuado (negativo) que la respuesta ante el aumento de los ingresos.

\section{Referencias}

Aguilar, A., Valles, Y. (2015). El ahorro en México: Evidencia en Hogares. Estudios Económicos CNBV, $3,41-72$.

Andersen, J. V., Nowak, A. (2013). An Introduction to Socio-Finance. Springer.

Banco de México. (2015). Indicadores basicos de tarjeta de credito: Datos a diciembre 2014. Recuperado el 16 de Noviembre de 2016, de Indicadores básicos de tarjetas de crédito: www.banxico.org.mx/sistemafinanciero/publicaciones/reporte-de-tasas-de-interes-efectivas-de-tarjetas-/reporte-tasas-interes-efectiv .html

Barberis, N. (2013). Thirty Years of Prospect Theory in Economics: A Review and Assessment. Journal of Economic Perspectives, 27, 173-196.

Borsch-Supan, A. (2003). Life-Cycle Savings and Public Policy: A Cross-National Study of Six Countries. Academic Press.

Borsch-Supan, A., Lusardi, A. (2003). Life-Cycle Savings and Public Policy: A Cross-National Study of Six Countries. Academic Press.

Bosworth, B., Burtless, G., Sabelhaus, J. (1991). The Decline in Saving: Evidence from hausehold Surveys (Vols. 1, 183-256). BrookingsPapers on Economic Activity.

Brown, F., Dominguez, L., Nava, I. (2014). Diferencias de género en los factores asociados al ahorro de los hogares en México. Estudios Demográficos y Urbanos, 29(2), 301-339.

Castro, P., Parsons, S. (2014). Modeling Agents Preferences Based on Prospect Theory. Multidisciplinary Workshop on Advances in Preference Handling, 32-36. Obtenido de Modeling Agents Preferences Based on Prospect Theory: www.aaai.org/ocs/index.php/WS/AAAIW14/paper/viewFile/8769/8278

Ceballos, O. (2018). Perfiles de ahorro y pago de deuda en el ciclo de vida de los hogares mexicanos. El Trimestre Económico, Vol. LXXV, número 338, 311-339.

Consejo Nacional de Inclusión Financiera. (2017). Reporte Nacional de Inclusión Financiera. Recuperado el 7 de Julio de 2018, de www. cnbv.gob.mx/Inclusi\%C3\%B3n/Documents/Reportes\%20de\%20IF/ Reporte\%20de\%20Inclusion\%20Financiera\%208.pdf

Copaciu, M., Croitoru, L., Mihaescu, F. (2010). Health Status and the Saving Behaviour of Pensioners. CERGE.

Cruz, H. (2017). Análisis del comportamiento del ahorro de los hogares en México y el impacto de características socioeconómicas: Un enfoque conductual. (Tesis de maestría). México: Escuela Superior de Economía. Instituto Politécnico Nacional.

Diamond, P., Vartiainen, H. (2007). Behavioral Economics and its applications. Princenton University Press.

Dietz, R., Haurin, D. (2003). The social and private micro-level consequences of homeownership (Vols. 54, 401-450). Journal of Urban Economics.

Engen, E., Gruber, J. (2001). Unemployment insurance and precautionary saving (Vols. 47, 545-579). Journal of Monetary Economics.

Finaly, R., Price, F. (2014). Household Saving in Australia. Reserve Bank of Australia, Research Discussion Paper.

Fisher, P. J., Montalto, C. P. (2011). Loss aversion and saving behavior: Evidence from the 2007 Survey of Consumer Finances. Journal of Family and Economic, Vol 32, 4-14.

Fredrickson, J. (2013). Prospect Theory and saving behaviors during the Great Recession: 2009 SCF Panel Survey Results. Journal of Behavioral Studies in Business, 6, 1-17.

Friedman, M. (1957). A Theory of the Consumption Function. Estados Unidos: Princeton University Press.

Gapinski, J. (1993). Questions on the Economics of Saving. (J. Gapinski, Ed.) The Economics of Saving. Springer.

Gasca, J. (2009). Geografía Regional: la región, la regionalización y el desarrollo regional en México. . México: Instituto de Geografía UNAM.

Góngora, J. P. (2013). Evaluación reciente del ahorro en México. Comercio Exterior., 63-1, 8-12.

Gonzalez, L., Ozcan, B. (2008). The Risk of Divorce and Household Saving Behavior (Vol. 3726). Institute for the Study of Labor, Discussion paper series. 
Grinstein-Weiss, M., Zhan, M. (2005). Educational Status and Savings Performance in Individual Development Accounts. Center for Social Development, Washington University.

Grinstein-Weiss, M., Sherraden, M., Zhan, Z. (2004). Saving Performance in Individual Development Accounts: Does Marital Status Matter? Center for Social Development, Washington University.

Instituto Nacional de Geografía y Estadística. (2017). Estadísticas a propósito del día de la familia Mexicana. Recuperado el Recuperado el 15 de mayo 2017, de www.inegi.org.mx/saladeprensa/aproposito/2017 /familia2017 ${ }_{N}$ al.pdf

Kennickell, A., Lusardi, A. (2003). Wealth Accumulation and the Importance of Precautionary Savings. Working paper, Dartmouth College.

Knoll, M. (2010). The Role of Behavioral Economics and Behavioral Decision Making in Americans' Retirement Savings Decisions (Vols. 70, No. 4). Social Security Bulletin.

Koszegi, B., Rabin, M. (November de 2006). A Model of Reference-Dependent Preferences. The Quarterly Journal of Economics, CXXI(4), 1133-1165.

Kovalerchuk, B., Vityaev, E. (2000). Data Mining in Finance. Springer.

Lee, S., Montalto, C., Park, M. (2000). The Effect of Family Life Cycle and Financial Management Practices on Household Saving Patterns (Vols. 1, 1, 79-93). Journal of Korean Home Economics Association English Edition.

Mendenhall, W., Scheaffer, R., Wackerly, D. (2010). Estadística matemática con aplicaciones. México: Cengage Learning.

Modigliani, F. (1986). Life cycle, thrift, and the wealth of nations (Vols. 76, issue 3). Estados Unidos: American Economic Review.

Modigliani, F., Ando, A. (1963). The "life cycle"hypothesis of saving aggregate implications and tests (Vols. 53, No. 1, Part 1, 55-84). Estados Unidos: American Economic Review.

Organización para la Cooperación y el Desarrollo Económico. (2017). Estudios Económicos de la OCDE. España: OCDE.

Ruiz, P., Ordaz, J. (Mayo-Agosto de 2011). Evolución reciente del empleo y el desempleo en México. Economía UNAM, 8(3), 91-105.

Secretaría de Desarrollo Agrario, Territorial y Urbano. (2015). Regionalización Funcional de México: Metodología. SEDATU.

Skinner, J. (1988). Risky income, life cycle consumption, and precautionary saving (Vols. 22, 237-255). Journal of Monetary Economics.

Smyth, D. (1993). Toward a theory of saving. (J. Gapinski, Ed.) The Economics of Saving. Springer.

Souleles, N. (1999). The Response of Household Consumption to Income Tax Refunds (Vols. 89(4), 947958). American Economic Review.

Székely, M. (1998). Monto y distribución del ahorro de los hogares en México (Vol. 65). El Trimestre Económico.

Thaler, R. (1990). Anomalies: Saving, fungibility, and mental accounts (Vols. 4, 193-205). The Journal of Economic Perspectives.

Villagómez, A. (1993). Los determinantes del ahorro en México (Vols. II-2, 305-327). México: Economía Mexicana.

Villagómez, A. (2008). El ahorro en México desde 1960: Estructura, evolución y determinación. Centro de Investigación y Docencia Económicas. 
REMEF (The Mexican Journal of Economics and Finance) 76

Anexos

Anexo 1. Cálculo por concepto de intereses

La ENIGH considera en partidas separadas el gasto corriente del hogar y las erogaciones financieras y de capital, dado que los pagos por intereses de las deudas adquiridas por el hogar representan una salida de recursos que no están disponibles para el ahorro se procedió a incorporarlos dentro del gasto corriente del hogar. Para ello, de acuerdo a (Székely, 1998) se considera el pago de intereses por concepto de pago de deudas por la adquisición de bienes raíces $L_{B}$, préstamos adquiridos con terceros $L_{T}$ y los pagos de tarjetas de crédito $L_{C}$. Cada uno de estos pagos comprende un monto correspondiente al pago de intereses (identificado por i) además de un monto correspondiente a la amortización de la deuda (identificado por M ).

$$
\begin{aligned}
& L_{B}=L_{B}^{i}+L_{B}^{M} \\
& \mathrm{~L}_{T}=L_{T}^{i}+L_{T}^{M} \\
& \mathrm{~L}_{C}=L_{C}^{i}+L_{C}^{M}
\end{aligned}
$$

El cálculo del componente que representa el pago de intereses considera que el pago monto de pago de cualquier deuda E puede expresarse por la siguiente expresión donde $(i * D)$ es el producto de la tasa de interés vigente por el valor de la deuda y $\frac{D}{N}$ el pago de una parte del principal (en este caso se asume que se divide de forma proporcional entre el número de años de contratación de la deuda).

$$
E=(i * D)+\frac{D}{N}
$$

La proporción que representa el pago de intereses sobre el monto pagado queda expresada por:

$$
\frac{(i * D)}{E}=\frac{(i * N)}{(i * N)+1}
$$

Al multiplicar dicha proporción por el monto pagado se obtendrá finalmente el monto de pago por intereses. Se consideró el siguiente valor para cada una de las variables:

\begin{tabular}{|l|c|c|}
\hline \multicolumn{1}{|c|}{ Tipo de deuda } & Tasa de interés & Años de contratación \\
\hline Bienes raíces & $10.77 \%^{4}$ & 20 \\
\hline Deudas con terceros & $3.18 \%^{5}$ & 2 \\
\hline Tarjeta de crédito & $23.90 \%^{6}$ & $6 / 12^{7}$ \\
\hline
\end{tabular}

Anexo 2. Características sociodemográficas de los perfiles de hogares

\begin{tabular}{|l|c|c|c|c|c|c|c|c|c|c|c|}
\hline Perfiles & 4 & $5 \_1$ & $5 \_2$ & 6 & 7 & 8 & 9 & $10 \_1$ & $10 \_2$ \\
\hline $\begin{array}{l}\text { Tipo de hogar } \\
\text { Unipersonal }\end{array}$ & $14.40 \%$ & $0.50 \%$ & $43.10 \%$ & $0.50 \%$ & $4.70 \%$ & $6.70 \%$ & $18.40 \%$ & - & - \\
\hline Corresidentes & $0.10 \%$ & $0.10 \%$ & $4.80 \%$ & $0.04 \%$ & - & - & $0.20 \%$ & - & $0.10 \%$ \\
\hline Nuclear & $68.20 \%$ & $88.10 \%$ & $19.50 \%$ & $87.30 \%$ & $66.60 \%$ & $70.00 \%$ & $64.80 \%$ & $21.70 \%$ & $2.60 \%$ \\
\hline $\begin{array}{l}\text { Ampliado y } \\
\text { compuesto }\end{array}$ & $17.20 \%$ & $11.30 \%$ & $32.50 \%$ & $12.20 \%$ & $28.80 \%$ & $23.30 \%$ & $16.60 \%$ & $78.30 \%$ & $97.40 \%$ \\
\hline
\end{tabular}


Revista Mexicana de Economía y Finanzas Nueva Época, Vol. 14 No. 1, (2019), pp. 57-77 DOI: http://dx.doi.org/10.21919/remef.v14i1.359

\begin{tabular}{|c|c|c|c|c|c|c|c|c|c|}
\hline \multicolumn{10}{|l|}{ Edad } \\
\hline Promedio años & 37.75 & 35.76 & 31 & 32.66 & 46.98 & 46.84 & 64.17 & 56.38 & 72.03 \\
\hline Menor 20 & - & $0.02 \%$ & $6.50 \%$ & $1.40 \%$ & - & - & - & - & - \\
\hline 20 a 24 & $2.50 \%$ & $4.50 \%$ & $18.30 \%$ & $14.60 \%$ & - & - & - & - & - \\
\hline 25 a 29 & $8.20 \%$ & $13.30 \%$ & $21.40 \%$ & $19.20 \%$ & - & - & - & - & - \\
\hline 30 a 34 & $14.40 \%$ & $20.60 \%$ & $15.90 \%$ & $20.80 \%$ & - & - & - & - & - \\
\hline 35 a 39 & $27.30 \%$ & $28.60 \%$ & $17.40 \%$ & $23.10 \%$ & - & - & - & - & - \\
\hline 40 a 44 & $47.60 \%$ & $33.00 \%$ & $20.50 \%$ & $21.10 \%$ & - & - & - & - & - \\
\hline 45 a 49 & - & - & - & - & $100.00 \%$ & $100.00 \%$ & - & - & - \\
\hline 50 a 54 & - & - & - & - & - & - & $20.70 \%$ & $40.20 \%$ & - \\
\hline 55 a 59 & - & - & - & - & - & - & $19.30 \%$ & $34.10 \%$ & - \\
\hline 60 a 64 & - & - & - & - & - & - & $16.30 \%$ & $25.70 \%$ & - \\
\hline 65 en adelante & - & - & - & - & - & - & $14.40 \%$ & - & $43.90 \%$ \\
\hline 70 a más & - & - & - & - & - & - & $29.20 \%$ & - & $56.10 \%$ \\
\hline \multicolumn{10}{|l|}{ Género } \\
\hline Mujer & $79.90 \%$ & $6.60 \%$ & $54.70 \%$ & $8.50 \%$ & $22.30 \%$ & $26.30 \%$ & $33.90 \%$ & $25.90 \%$ & $32.10 \%$ \\
\hline Hombre & $20.10 \%$ & $93.40 \%$ & $45.30 \%$ & $91.50 \%$ & $77.70 \%$ & $73.70 \%$ & $66.10 \%$ & $74.10 \%$ & $67.90 \%$ \\
\hline \multicolumn{10}{|l|}{ Escolaridad } \\
\hline Sin instrucción & $1.10 \%$ & $1.30 \%$ & $0.30 \%$ & $2.50 \%$ & $9.80 \%$ & - & $14.50 \%$ & $6.40 \%$ & $29.00 \%$ \\
\hline Primaria & $21.80 \%$ & $21.60 \%$ & $10.00 \%$ & $25.30 \%$ & $90.20 \%$ & - & $40.80 \%$ & $49.40 \%$ & $55.60 \%$ \\
\hline Secundaria & $45.50 \%$ & $36.10 \%$ & $20.80 \%$ & $43.40 \%$ & - & $47.90 \%$ & $19.50 \%$ & $26.40 \%$ & $8.10 \%$ \\
\hline Preparatoria & $14.90 \%$ & $23.00 \%$ & $21.70 \%$ & $19.50 \%$ & - & $24.70 \%$ & $8.10 \%$ & $9.10 \%$ & $2.70 \%$ \\
\hline Superior & $14.10 \%$ & $14.70 \%$ & $41.30 \%$ & $9.10 \%$ & - & $23.10 \%$ & $14.60 \%$ & $6.80 \%$ & $4.30 \%$ \\
\hline Posgrado & $2.60 \%$ & $3.40 \%$ & $5.90 \%$ & $0.40 \%$ & - & $4.40 \%$ & $2.60 \%$ & $2.00 \%$ & $0.20 \%$ \\
\hline $\begin{array}{c}\text { Presencia de } \\
\text { menores }\end{array}$ & $56.90 \%$ & $81.00 \%$ & $23.50 \%$ & $78.50 \%$ & $53.10 \%$ & $45.70 \%$ & 一 & $100.00 \%$ & $100.00 \%$ \\
\hline \multicolumn{10}{|l|}{ Estado civil } \\
\hline Unión libre & - & - & - & $100 \%$ & $19.10 \%$ & $11.60 \%$ & $5.80 \%$ & $15.10 \%$ & $7.60 \%$ \\
\hline Casado & - & $100 \%$ & - & - & $61.60 \%$ & $63.70 \%$ & $52.80 \%$ & $60.20 \%$ & $53.30 \%$ \\
\hline Separado & $66.40 \%$ & - & - & - & $6.80 \%$ & $8.30 \%$ & $7.70 \%$ & $7.60 \%$ & $4.80 \%$ \\
\hline Divorciado & $22.20 \%$ & - & - & - & $1.20 \%$ & $6.50 \%$ & $3.80 \%$ & $1.60 \%$ & $0.10 \%$ \\
\hline Viudo & $11.40 \%$ & - & - & - & $6.90 \%$ & $2.40 \%$ & $22.50 \%$ & $13.00 \%$ & $33.60 \%$ \\
\hline Soltero & - & - & $100 \%$ & - & $4.50 \%$ & $7.50 \%$ & $7.40 \%$ & $2.40 \%$ & $0.70 \%$ \\
\hline
\end{tabular}

Fuente: Elaboración propia con información de ENIGH 2014

\section{Incidencia factores de incertidumbre}

\begin{tabular}{|l|c|c|c|c|c|c|c|c|c|c|}
\hline $\begin{array}{l}\text { Perfiles } \\
\text { Tipo de hogar }\end{array}$ & 5 & $5 \_1$ & $5 \_2$ & 6 & 7 & 8 & 9 & $10 \_1$ & $10 \_2$ \\
\hline Problema de salud & $34.4 \%$ & $30.6 \%$ & $25.8 \%$ & $31.5 \%$ & $34.5 \%$ & $31.0 \%$ & $37.7 \%$ & $35.9 \%$ & $46.0 \%$ \\
\hline Autoempleo & $15.5 \%$ & $17.0 \%$ & $10.7 \%$ & $13.4 \%$ & $31.9 \%$ & $23.1 \%$ & $24.9 \%$ & $27.3 \%$ & $24.2 \%$ \\
\hline Desempleo & $0.8 \%$ & $3.1 \%$ & $2.9 \%$ & $2.0 \%$ & $2.3 \%$ & $4.1 \%$ & $2.7 \%$ & $3.9 \%$ & $0.8 \%$ \\
\hline Subempleo & $16.4 \%$ & $6.9 \%$ & $17.6 \%$ & $7.3 \%$ & $18.4 \%$ & $12.7 \%$ & $14.6 \%$ & $14.6 \%$ & $15.0 \%$ \\
\hline Personas ocupadas* & $48.5 \%$ & $40.6 \%$ & $64.8 \%$ & $40.9 \%$ & $49.2 \%$ & $49.4 \%$ & $47.9 \%$ & $39.4 \%$ & $34.4 \%$ \\
\hline
\end{tabular}

*Proporción media personas ocupadas que reciben ingreso monetario del total de integrantes. Fuente: Elaboración propia con información de ENIGH 2014

\section{Propiedad de una vivienda}

\begin{tabular}{|l|c|c|c|c|c|c|c|c|c|}
\hline Perfiles & 4 & $5 \_1$ & $5 \_2$ & 6 & 7 & 8 & 9 & $10 \_1$ & $10 \_2$ \\
\hline Tipo de hogar \\
\hline Pagada & $37.4 \%$ & $44.3 \%$ & $22.6 \%$ & $37.1 \%$ & $64.9 \%$ & $58.3 \%$ & $78.2 \%$ & $76.2 \%$ & $85.7 \%$ \\
\hline Pagando & $9.6 \%$ & $16.2 \%$ & $8.2 \%$ & $8.3 \%$ & $5.1 \%$ & $17.6 \%$ & $4.7 \%$ & $5.0 \%$ & $2.0 \%$ \\
\hline
\end{tabular}

Fuente: Elaboración propia con información de ENIGH 2014 